\title{
EMBEDDED MINIMAL SURFACES AND TOTAL CURVATURE OF CURVES IN A MANIFOLD
}

\author{
Jaigyoung Choe and Robert Gulliver
}

\begin{abstract}
Let $M^{n}$ be an $n$-dimensional complete simply connected Riemannian manifold with sectional curvature bounded above by a nonpositive constant $-\kappa^{2}$. It is proved that every branched minimal surface in $M$ bounded by a smooth Jordan curve $\Gamma$ with total curvature $\leq 4 \pi+\kappa^{2} \inf _{p \in M}$ Area $(p \nVdash \Gamma)$ is embedded. $p \times \Gamma$ denotes the geodesic cone over $\Gamma$ with vertex $p$. It follows that a Jordan curve $\Gamma$ in $M^{3}$ with total curvature $\leq 4 \pi+\kappa^{2} \inf _{p \in M}$ Area $(p \times \Gamma)$ is unknotted. In the hemisphere $\mathbf{S}_{+}^{n}$, we prove the embeddedness of any minimal surface whose boundary curve has total curvature $\leq 4 \pi-\sup _{p \in \mathbf{S}_{+}^{n}} \operatorname{Area}(p \times \Gamma)$.
\end{abstract}

\section{Introduction}

After the formidable problem of Plateau in Euclidean $\mathbf{R}^{n}$ was settled by Douglas and Radó, mathematicians' attention was drawn to the uniqueness and embeddedness of their solutions (see [D] and [R1].). The first uniqueness result was obtained by Radó ([R2], p. 100). He proved that if a simple closed curve $\Gamma \subset \mathbf{R}^{3}$ has a one-to-one projection onto the boundary of a convex region $R \subset$ $\mathbf{R}^{2}$, then $\Gamma$ bounds a unique minimal disk. In fact any minimal surface bounded by $\Gamma$ is a graph over $R$, and hence is simply connected and embedded. Later Nitsche [N2] showed that if $\Gamma$ is analytic with total curvature $\leq 4 \pi$, then $\Gamma$ bounds exactly one minimal disk.

The embeddedness of the minimal disk bounded by a Jordan curve $\Gamma$ was first obtained by Gulliver and Spruck [GS] under the assumption that $\Gamma$ has total curvature $\leq 4 \pi$ and is extreme (that is, it lies on the boundary of a convex set). In the same paper, they conjectured that either condition alone would be sufficient for the embeddedness of an area-minimizing disk. Moreover Nitsche himself asked whether his unique solution is free of self-intersection ([N3], esp. p. 463). Indeed Tomi-Tromba [TT], Almgren-Simon [AS], and Meeks-Yau [MY] derived the embeddedness of a minimal disk bounded by an extreme $\Gamma$; $[\mathrm{MY}]$ proved embeddedness of any area-minimizing disk. But the sufficiency of the total curvature condition alone, when $\Gamma$ is not assumed to be extreme, remained open for 25 years.

Received July 3, 2001.

Work of the first author supported in part by KOSEF 2000-2-10200-002-5 and RIM-GARC. Work of the second author supported in part by the University of Melbourne and Monash University, Australia. 
Finally, in a very recent paper, Ekholm, White, and Wienholtz [EWW] ingeniously proved the embeddedness of any minimal surface bounded by a curve $\Gamma$ in $\mathbf{R}^{n}$ with total curvature $\leq 4 \pi$. Their idea is based on the following observations.

(i) The logarithm of the distance function $\rho(x)=d(x, p)$ in $\mathbf{R}^{n}$ is a fundamental solution of the Laplacian on a two-dimensional plane through $p$. Similarly, $\log \rho(x)$ is harmonic on a cone $p \times \Gamma$ over $\Gamma$ with vertex $p$. By contrast, $\log \rho(x)$ is strictly subharmonic on a nonplanar (branched) minimal surface in $\mathbf{R}^{n}$. This part of their proof is intimately related to the well-known monotonicity formula.

(ii) By the Gauss-Bonnet theorem, $2 \pi$ times the density at $p$ of the cone $p \times \Gamma$ is at most the total curvature of $\Gamma$.

In this paper we extend the Ekholm-White-Wienholtz result to minimal surfaces in an $n$-dimensional Riemannian manifold $M$ with sectional curvature bounded above by a nonpositive constant $-\kappa^{2}$. The two observations above can be appropriately generalized for these purposes. Thus, it is proved that if $\Gamma$ is a Jordan curve in $M^{n}$ with total curvature

$$
\mathcal{C}_{\mathrm{tot}}(\Gamma):=\int_{\Gamma}|\vec{k}| d s \leq 4 \pi+\kappa^{2} \inf _{p \in M} \operatorname{Area}(p \circledast \Gamma),
$$

then every branched minimal surface bounded by $\Gamma$ is embedded (Theorem 3.) More precisely, the infimum of area is taken only over geodesic cones with vertex lying in the convex hull $\mathcal{H}_{\mathrm{cvx}}(\Gamma)$ of $\Gamma$. In the presence of variable ambient curvatures, a key point is the introduction of a new metric of constant Gauss curvature on $p \times \Gamma$.

A similar theorem is also proved for minimal surfaces in the hemisphere $\mathbf{S}_{+}^{n}$, using $\kappa^{2}=-1$ (see Theorem 1.) This case is simpler, since only one metric

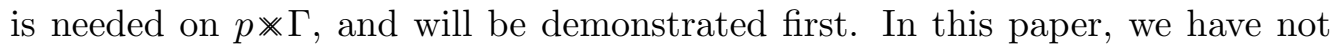
carried out the extension of our results to continuous Jordan curves, as was done in $[\mathrm{M}]$ and in $[\mathrm{EWW}]$.

As in [EWW], our theorem has a topological implication: any Jordan curve in $M^{3}$ with total curvature $\leq 4 \pi+\kappa^{2} \inf _{p \in M} \operatorname{Area}(p \times \Gamma)$ is unknotted. This appears to be a new extension of the Fáry-Milnor theorem, which showed that any knotted curve in $\mathbf{R}^{3}$ has total curvature greater than $4 \pi[\mathrm{F}],[\mathrm{M}]$. Brickell and Hsiung proved our unknotting result for the case when $M^{3}$ is the hyperbolic space of constant sectional curvature $-\kappa^{2}$ (see Theorem 4 of $[\mathrm{BH}]$.) It should also be mentioned that Schmitz $[\mathrm{S}]$ and Alexander-Bishop [AB] obtained the unknottedness of a Jordan curve with total curvature $\leq 4 \pi$ in a simply connected Riemannian 3-manifold of nonpositive sectional curvature, which is the case $\kappa=0$ of our Theorem 4. Alexander and Bishop also noted that the minimum total curvature among knotted curves in any non-positively curved 3-manifold is exactly $4 \pi$. But for the case of a manifold $M^{3}$ with sectional curvature $\leq-\kappa^{2}<$ 0 our hypothesis on the total curvature of $\Gamma$ is weaker, and more natural, since there are no homotheties, and thus no scaling, in $M^{3}$. 
One indication of the naturalness of our hypothesis, that a curve $\Gamma \subset M^{n}$ have total curvature $\leq 4 \pi+\kappa^{2} \inf _{p \in M}$ Area $(p \times \Gamma)$, is the fact that every closed curve in $M$ has total curvature at least $2 \pi+\kappa^{2} \inf _{p \in M}$ Area $(p \times \Gamma)$.

\section{Embeddedness of Minimal Surfaces in the Hemisphere}

Recall that in the open hemisphere $\mathbf{S}_{+}^{n}:=\left\{x \in \mathbf{R}^{n+1}:|x|=1, x_{n+1}>0\right\}$, any two points $p, q$ may be connected by a unique geodesic, namely the shorter arc of the unique great circle of $\mathbf{S}^{n}$ passing through $p$ and $q$. In particular, for any point $p \in \mathbf{S}_{+}^{n}$ and any immersed curve $\Gamma$ in $\mathbf{S}_{+}^{n}$, we may define the geodesic cone $p \times \Gamma$ to be the union of the geodesic segments from $p$ to $q$, over all $q \in \Gamma$. The smallest closed subset of $\mathbf{S}_{+}^{n}$ which contains a set $S \subset \mathbf{S}_{+}^{n}$ and contains the geodesic segment between any two of its points is the convex hull of $S$, and will be written as $\mathcal{H}_{\mathrm{cvx}}(S)$. Observe that, since $\mathbf{S}_{+}^{n}$ is a space form, $\mathcal{H}_{\mathrm{cvx}}(S)$ may also be described as the intersection of all closed hemispheres containing $S$. It follows that if $\Sigma$ is an immersed minimal surface in $\mathbf{S}_{+}^{n}$ with compact closure, whose boundary $\partial \Sigma \subset S$, then $\Sigma \subset \mathcal{H}_{\mathrm{cvx}}(S)$.

Definition 1. Define the maximum cone area of a curve $\Gamma \subset \mathbf{S}_{+}^{n}$ as

$$
\overline{\mathcal{A}}(\Gamma):=\sup _{p \in \mathcal{H}_{\mathrm{cvx}}(\Gamma)} \operatorname{Area}(p \circledast \Gamma) .
$$

Theorem 1. Let $\Gamma$ be a $C^{2}$ Jordan curve in the $n$-dimensional hemisphere $\mathbf{S}_{+}^{n}$. Suppose $\Sigma^{2}$ is a branched minimal surface, having compact closure in $\mathbf{S}_{+}^{n}$ and boundary $\Gamma=\partial \Sigma$. If the total curvature of $\Gamma$ satisfies

$$
\mathcal{C}_{\text {tot }}(\Gamma):=\int_{\Gamma}|\vec{k}| d s \leq 4 \pi-\overline{\mathcal{A}}(\Gamma)
$$

then $\bar{\Sigma}$ is an embedding.

In the definition of $\mathcal{C}_{\text {tot }}, \vec{k}$ denotes the curvature vector of $\Gamma$. If a point traverses $\Gamma$ with unit speed, then its acceleration vector in $\mathbf{S}_{+}^{n}$ coincides with $\vec{k}$. A branched minimal surface is one which may fail to be immersed at a discrete set of singularities, which are all branch points; see Definition 2 below.

We shall give the proof of Theorem 1 at the end of this section.

Theorem 1 has an interesting topological consequence: a new extension of the Fáry-Milnor Theorem. The Fáry-Milnor Theorem showed that a knotted curve in Euclidean $\mathbf{R}^{3}$ has total curvature at least $4 \pi([\mathrm{F}],[\mathrm{M}]$.) The next theorem is what we feel is an appropriate analogue of the Fáry-Milnor Theorem, when $\mathbf{R}^{3}$ is replaced by $\mathbf{S}_{+}^{3}$. We are not aware of any previous results on total curvature of knots in $\mathbf{S}_{+}^{3}$. Note that the bound required from above on total curvature in this theorem may be zero or even negative, in which case the theorem fails. However, in Example 1 below, we shall show that the bound is sharp, in the sense that there are knotted curves in $\mathbf{S}_{+}^{3}$ for which the total curvature is close to zero and the maximum cone area is close to $4 \pi$. 
Theorem 2. If $\Gamma$ is a $C^{2}$ Jordan curve in $\mathbf{S}_{+}^{3}$, with total curvature

$$
\int_{\Gamma}|\vec{k}| d s \leq 4 \pi-\overline{\mathcal{A}}(\Gamma)
$$

then $\Gamma$ is unknotted.

Proof. It follows from a theorem of Morrey that there is a smooth branched immersion of the disk into $\mathbf{S}_{+}^{3}$ with boundary $\Gamma$, having smallest area among surfaces of the type of the disk. Morrey's result [Mo] requires the ambient manifold $M^{3}$ to be complete and homogeneously regular. Recall that homogeneous regularity is an appropriately weak version of bounded geometry; see [Mo]. In order to apply Morrey's result to our case, we first need to construct a complete and homogeneously regular manifold $M^{3}$ in place of $\mathbf{S}_{+}^{3}$. Since $\Gamma$ is compact, it lies in a closed geodesic ball $B_{R} \subset \mathbf{S}_{+}^{3}$ of radius $R<\pi / 2$, with center the point of rotational symmetry $p_{0} \in \mathbf{S}_{+}^{3}$. We extend $B_{R}$ isometrically to a Riemannian manifold $M$ diffeomorphic to $\mathbf{R}^{3}$, with a rotationally symmetric metric, so that $M$ is complete and homogeneously regular, and the distance balls $B_{r}$ of $M$ from $p_{0}$ are convex, $0<r<\infty$. To make $M$ homogeneously regular, we may choose the metric to have e. $g$. the cylindrical form $\mathbf{S}_{b}^{2} \times\left[r_{1}, \infty\right)$ outside a compact set. Morrey's result shows that there is a smooth branched immersion of the disk into $M$ with boundary $\Gamma$, having smallest area among surfaces of the type of the disk. Write its closed image as $\Sigma$. Since $\Sigma$ is compact, it lies in $B_{r_{0}}$ for some $r_{0}$, and since each $B_{r}$ is convex, $R \leq r<\infty$, we see by the maximum principle that $\Sigma \subset B_{R}$. Therefore $\Sigma \subset \mathbf{S}_{+}^{3}$.

According to Theorem 1, this area-minimizing disk must be an embedding of the disk into $\mathbf{S}_{+}^{3}$ with boundary $\Gamma$; this shows that $\Gamma$ is unknotted.

An alternative proof of Theorem 2 may be given for a real-analytic curve $\Gamma$, and by approximation for a $C^{2}$ curve which satisfies $\mathcal{C}_{\text {tot }}(\Gamma)<4 \pi-\overline{\mathcal{A}}(\Gamma)$. The alternate proof requires Theorem 1 only for an immersed minimal surface $\Sigma$, and cites the result that the area-minimizing branched immersion from the disk into $\mathbf{S}_{+}^{3}$ with boundary $\Gamma$ must be an immersion up to the boundary (see $[A],[G]$ and [GL].)

Example 1. With this example, we shall show that the hypothesis

$$
\mathcal{C}_{\text {tot }}(\Gamma) \leq 4 \pi-\overline{\mathcal{A}}(\Gamma)
$$

of Theorems 1 and 2 (which may appear very strong from a certain point of view) is actually sharp.

Let $\Gamma_{0}$ be the double cover of the circle of some radius $R<\pi / 2$ in a totally geodesic $\mathbf{S}_{+}^{2} \subset \mathbf{S}_{+}^{3}$, with center at $p_{0}$. This example is a family of $(2,2 m+1)$ torus knots $\Gamma_{\eta}$ in $\mathbf{S}_{+}^{3}, \eta>0$, for any fixed positive integer $m$, such that the $C^{2}$ distance between $\Gamma_{\eta}$ and $\Gamma_{0}$ as parameterized curves approaches zero as $\eta \rightarrow 0$, and such that

$$
\mathcal{C}_{\text {tot }}\left(\Gamma_{\eta}\right)<4 \pi-\overline{\mathcal{A}}\left(\Gamma_{\eta}\right)+\eta
$$


To be specific, we might choose $\Gamma_{\eta}$ to lie on the boundary of the tubular neighborhood of $\Gamma_{0}$ at a radius which tends to 0 as $\eta \rightarrow 0$.

We first compute the geometric invariants of $\Gamma_{0}$. Its length is $4 \pi \sin R$, and its curvature is constant: $|\vec{k}| \equiv \cot R$. Thus, $\mathcal{C}_{\text {tot }}\left(\Gamma_{0}\right)=4 \pi \cos R$. The maximum cone area $\overline{\mathcal{A}}\left(\Gamma_{0}\right)=2 \cdot 2 \pi \int_{0}^{R} \sin r d r=4 \pi(1-\cos R)$ is achieved by the double cover of the totally geodesic disk of radius $R$, since this disk is the convex hull $\mathcal{H}_{\mathrm{cvx}}\left(\Gamma_{0}\right)$ of $\Gamma_{0}$. Thus, equality holds in hypothesis $(1)$ for $\Gamma_{0}: \mathcal{C}_{\text {tot }}\left(\Gamma_{0}\right)=$ $4 \pi-\overline{\mathcal{A}}\left(\Gamma_{0}\right)$. But both of the geometric invariants $\overline{\mathcal{A}}(\Gamma)$ and $\mathcal{C}_{\text {tot }}(\Gamma)$ are continuous as $\Gamma$ varies in $C^{2}$. We find therefore $\mathcal{C}_{\text {tot }}\left(\Gamma_{\eta}\right)<4 \pi-\overline{\mathcal{A}}\left(\Gamma_{\eta}\right)+\eta$, as claimed, showing that hypothesis (1) is sharp. 0 .

If we choose $R=R(\eta) \rightarrow \pi / 2$, then we may obtain further that $\mathcal{C}_{\text {tot }}\left(\Gamma_{\eta}\right) \rightarrow$

Propositions 1 and 2 below will form the core of the proof of Theorem 1 .

For the rest of this section, we shall write $G(r):=\log \tan (r / 2)$ for the Green's function of the two-dimensional sphere. Choose a point $p \in \mathbf{S}_{+}^{n}$, and for all $x \in \mathbf{S}_{+}^{n}$, define $\rho(x):=d(x, p)$, the distance measured in $\mathbf{S}_{+}^{n}$.

Lemma 1. Let $N^{2}$ be a two-dimensional manifold immersed in $\mathbf{S}_{+}^{n}$. Then except at $p$,

$$
\triangle_{N} G(\rho)=2 \frac{\cos \rho}{\sin ^{2} \rho}\left(1-\left|\nabla_{N} \rho\right|^{2}\right)+\frac{d \rho(\vec{H})}{\sin \rho},
$$

where $\vec{H}$ denotes the mean curvature vector of $N$.

Proof. In $\mathbf{S}_{+}^{n}$, the Hessian of the distance function is $\bar{\nabla}^{2} \rho=\cot \rho(g-\bar{\nabla} \rho \otimes \bar{\nabla} \rho)$, where $g$ is the metric tensor of $\mathbf{S}_{+}^{n}$. The trace formula states that

$$
\triangle_{N} G=\sum_{\alpha=1}^{2} \bar{\nabla}^{2} G\left(e_{\alpha}, e_{\alpha}\right)+d G(\vec{H}),
$$

where $\left\{e_{1}, e_{2}\right\}$ is an orthonormal basis for the tangent plane to $N$. These formulas are well known (see e. g. [CG2], pp. 172, 174.) Choosing $\left\{e_{1}, e_{2}\right\}$ with $d \rho\left(e_{2}\right)=0$ and $d \rho\left(e_{1}\right)=\left|\nabla_{N} \rho\right|$, we have

$$
\bar{\nabla}^{2} G\left(e_{1}, e_{1}\right)=\frac{\cos \rho}{\sin ^{2} \rho}\left(1-2 d \rho\left(e_{1}\right)^{2}\right)
$$

and

The conclusion follows.

$$
\bar{\nabla}^{2} G\left(e_{2}, e_{2}\right)=\frac{\cos \rho}{\sin ^{2} \rho}
$$

Definition 2. Let $\Omega$ be a Riemann surface, $k$ a positive integer. A mapping $f: \Omega \rightarrow M^{n}$ has a branch point of order $k$ at $w_{0} \in \Omega$ if its complex first partial derivative $f_{w}:=\frac{1}{2}\left(f_{u}-i f_{v}\right)$ satisfies $\lim _{w \rightarrow w_{0}}\left[f_{w}(w)\left(w-w_{0}\right)^{-k}\right]=\vec{a} \in \mathbf{C}^{n} \backslash\{0\}$. Here $u$ and $v$ are the real and imaginary parts of the local complex variable $w \in \Omega$, and $i=\sqrt{-1}$. 
A branched minimal surface $f: \Omega \rightarrow M^{n}$ is a conformally parameterized harmonic mapping. By abuse of language, we shall also refer to the image $\Sigma=f(\Omega)$ of $f$ as a branched minimal surface.

It may be shown that each point of a branched minimal surface either is a branch point or has an immersed neighborhood; moreover, the real and imaginary parts of the complex vector $\vec{a}$ in the definition of a branch point are orthogonal and have equal length (see $[\mathrm{HH}]$ ). The importance of branched minimal surfaces stems from the fact that the solution of Plateau's problem for a minimal surface of a given topological type in $\mathbf{R}^{n}$ or in $M^{n}$ is not an immersion in general, but only a branched immersion. Solutions to this variational problem are necessarily immersions only when $n=3$ ([A], [G], [GL]), or when the boundary curve meets hyperplanes of $\mathbf{R}^{n}$ in at most five points ([R2], pp. 34-35), or when the topological type is not prescribed ([Fed], [HS].)

The following lemma describes the effect of branch points on area and divergence-theorem computations on a branched minimal surface. Part (b) shows that if $p \notin \Sigma$, then there is no effect on the integral of $\triangle_{\Sigma} G$. The conclusion of part (a) may be interpreted to say that for some purposes, $\Sigma$ acts like the $(k+1)$-fold cover of a smooth surface near a branch point of order $k$.

Lemma 2. Let $\Sigma=f(\Omega)$ be a branched minimal surface in a Riemannian manifold $M$.

(a) Let $p=f\left(w_{0}\right), w_{0} \in \bar{\Omega}$, be a branch point of $\Sigma$ of order $k$. If $\nu_{\Sigma}$ is the unit normal vector to $\Sigma \cap \partial B_{\varepsilon}(p)$ tangent to $\Sigma$ and pointing towards $p$, then as $\varepsilon \rightarrow 0, \nu_{\Sigma} \rightarrow-\bar{\nabla} \rho$ uniformly on $\Sigma \cap \partial B_{\varepsilon}(p)$. After rescaling to unit radius, the curve $\Sigma \cap \partial B_{\varepsilon}(p)$ converges in $C^{1}$ norm to the constant-speed $(k+1)$-fold cover (resp. half of the constant-speed $(k+1)$-fold cover) of a great circle in the unit sphere of $T_{p}(M)$, if $w_{0} \in \Omega$ (resp. $w_{0} \in \partial \Omega$ ). Moreover, if $w_{0} \in \partial \Omega$ and $f$ maps $\partial \Omega$ monotonically to a $C^{2}$ curve $\Gamma$, then $k$ is even.

(b) If $p \notin \Sigma$, then

$$
\int_{\Sigma} \triangle_{\Sigma} G d A=\int_{\partial \Sigma} \nu_{\Sigma} \cdot \bar{\nabla} G d s
$$

where $\nu_{\Sigma}$ is the outward unit normal vector to $\partial \Sigma$ tangent to $\Sigma$.

Proof. Choose local conformal coordinates for $\Omega$ near $w_{0}$ and Riemannian normal coordinates for $M$ at $p$. Write $\vec{a}=\lim _{w \rightarrow w_{0}}\left(\left(w-w_{0}\right)^{-k} f_{w}(w)\right)=: \vec{b}+i \vec{c}$, where the real vectors $\vec{b}$ and $\vec{c}$ are orthogonal and have the same length (see [HH].) Then as $w \rightarrow w_{0}$, the tangent plane to $\Sigma$ at $f(w)$ converges to the plane in $T_{p}(M)$ spanned by $\vec{b}$ and $\vec{c}$. Integration shows that $f(w)-f\left(w_{0}\right)$ is the real part of $\frac{2}{k+1} \vec{a}\left(w-w_{0}\right)^{k+1}$, modulo a term which tends to zero faster than $\left|w-w_{0}\right|^{k+1}$. The parity of $k$ at a boundary branch point was shown in [N1], p. 332. The conclusions of part (a) follow.

To prove part (b), we apply part (a) to each branch point $q_{i}=f\left(w_{i}\right)$ of $\Sigma$, $1 \leq i \leq m$. The divergence theorem on $\Sigma \backslash \cup_{i=1}^{m} B_{\varepsilon}\left(q_{i}\right)$ leads to the $m$ additional 
boundary terms

$$
\int_{\Sigma \cap \partial B_{\varepsilon}\left(q_{i}\right)} \nu_{\Sigma} \cdot \bar{\nabla} G d s .
$$

Since $p \notin \Sigma, \nu_{\Sigma} \cdot \bar{\nabla} G$ is uniformly bounded in a neighborhood of $q_{i}$, while the length of $\Sigma \cap \partial B_{\varepsilon}\left(q_{i}\right)$ approaches 0 by part (a), so these additional boundary terms tend to 0 as $\varepsilon \rightarrow 0$.

Corollary 1. If $\Sigma^{2}$ is a branched minimal surface in $\mathbf{S}_{+}^{n}$, then $G(\rho(x))=$ $\log \tan (\rho(x) / 2)$ is subharmonic on $\Sigma$. If $C$ is the cone $p \times \partial \Sigma$ over the pole $p$ of the distance function $\rho$, then $G(\rho)$ is harmonic on $C$, except at $p$.

Proof. Since $\rho$ is a distance function in $\mathbf{S}_{+}^{n},\left|\nabla_{\Sigma} \rho\right| \leq 1$, while on the cone, since the $\mathbf{S}_{+}^{n}$-gradient $\bar{\nabla} \rho$ is tangent to $C,\left|\nabla_{C} \rho\right| \equiv 1$. The mean curvature vector of $\Sigma$ vanishes, and the mean curvature vector of $C$ is orthogonal to the gradient $\bar{\nabla} \rho$. Lemmas 1 and $2(\mathbf{a})$ now imply that $\triangle_{\Sigma} G(\rho) \geq 0$ and $\triangle_{C} G(\rho) \equiv 0$, except at $p$.

If $p \in \Sigma$, then the outward normal derivative of $G(\rho)$ on $\partial B_{\varepsilon}(p) \cap \Sigma$ approaches $+\infty$ as $\varepsilon \rightarrow 0$ (if $p$ is a branch point of $\Sigma$, Lemma 2(a) will be useful here), which implies that $G$ is subharmonic everywhere on $\Sigma$.

For a 2-dimensional immersed Lipschitz surface, or a branched surface, $N^{2} \subset$ $\mathbf{S}_{+}^{n}$, we define the density of $N$ at $q$ to be the limit

$$
\Theta_{N}(q):=\lim _{\varepsilon \rightarrow 0} \frac{\operatorname{Area}\left(N \cap B_{\varepsilon}(q)\right)}{\pi \varepsilon^{2}} .
$$

Here, $B_{\varepsilon}(q)$ is the geodesic ball of $\mathbf{S}_{+}^{n}$ with spherical radius $\varepsilon$, centered at $q$. Note that when $N$ is smooth or a cone, we may also compute the density in terms of lengths:

$$
\Theta_{N}(q)=\lim _{\varepsilon \rightarrow 0} \frac{L\left(N \cap \partial B_{\varepsilon}(q)\right)}{2 \pi \varepsilon} .
$$

Of course, the same limit is also obtained if the denominators in these two quotients are replaced by the spherical area $2 \pi(1-\cos \varepsilon)$ and spherical length $2 \pi \sin \varepsilon$, respectively. Observe that if $N$ is a smoothly immersed submanifold and has a self-intersection at $p \in \mathbf{S}_{+}^{n}$, then $\Theta_{N}(p) \geq 2$. Also note that if $p$ is a branch point of $N$ of order $k$, then $\Theta_{N}(p) \geq k+1$ (see the proof of Lemma $2(\mathbf{a})$.)

Proposition 1 (Density Comparison). Let $\Gamma$ be a $C^{2}$ immersed closed curve in $\mathbf{S}_{+}^{n}$. Choose $p \in \mathbf{S}_{+}^{n} \backslash \Gamma$. If $\Sigma^{2}$ is a branched minimal surface in $\mathbf{S}_{+}^{n}$ with boundary $\partial \Sigma=\Gamma$, and $C$ is the cone $p \times \Gamma$ over $p$, then their densities at $p$ satisfy the inequality

$$
\Theta_{\Sigma}(p)<\Theta_{C}(p)
$$

unless $\Sigma$ is totally geodesic. 
Proof. By Corollary 1, we have $\triangle_{\Sigma} G(\rho) \geq 0$ and $\triangle_{C} G(\rho) \equiv 0$, where $G(\rho(x)):=$ $\log \tan (\rho(x) / 2)$ and $\rho(x):=d(x, p)$. For small $\varepsilon>0$, write $C_{\varepsilon}:=C \backslash B_{\varepsilon}(p)$, and similarly $\Sigma_{\varepsilon}$. Then the boundary of $\Sigma_{\varepsilon}$ is $\Gamma \cup\left(\Sigma \cap \partial B_{\varepsilon}(p)\right)$. Let $\nu_{\Sigma}\left(\nu_{C}\right.$, respectively) be the outward unit normal vector tangent to $\Sigma_{\varepsilon}$ at $\partial \Sigma_{\varepsilon}$ (to $C_{\varepsilon}$ at $\partial C_{\varepsilon}$, resp.). Then

$0 \leq \int_{\Sigma_{\varepsilon}} \triangle_{\Sigma} G(\rho) d A=\int_{\partial \Sigma_{\varepsilon}} \nu_{\Sigma} \cdot \bar{\nabla} G d s=\int_{\Sigma \cap \partial B_{\varepsilon}(p)} \frac{\nu_{\Sigma} \cdot \bar{\nabla} \rho}{\sin \varepsilon} d s+\int_{\Gamma} \frac{\nu_{\Sigma} \cdot \bar{\nabla} \rho}{\sin \rho} d s$.

Along the small boundary component $\Sigma \cap \partial B_{\varepsilon}(p)$, as $\varepsilon \rightarrow 0, \nu_{\Sigma} \cdot \bar{\nabla} \rho \rightarrow-1$ uniformly, and

$$
\frac{L\left(\Sigma \cap \partial B_{\varepsilon}(p)\right)}{2 \pi \sin \varepsilon} \rightarrow \Theta_{\Sigma}(p) .
$$

Along $\Gamma, \quad \nu_{\Sigma} \cdot \bar{\nabla} \rho \leq \nu_{C} \cdot \bar{\nabla} \rho$. Hence as $\varepsilon \rightarrow 0$, we find

$$
2 \pi \Theta_{\Sigma}(p) \leq \int_{\Gamma} \frac{\nu_{C} \cdot \bar{\nabla} \rho}{\sin \rho} d s .
$$

Similarly, along $C \cap \partial B_{\varepsilon}(p)$, we have $\nu_{C} \equiv-\bar{\nabla} \rho$. After applying the divergence theorem to the vector field $\nabla_{C} G(\rho)$ on $C_{\varepsilon}$, we find

$$
2 \pi \Theta_{C}(p)=\int_{\Gamma} \frac{\nu_{C} \cdot \bar{\nabla} \rho}{\sin \rho} d s .
$$

This implies $\Theta_{\Sigma}(p) \leq \Theta_{C}(p)$. If equality holds, then $\triangle_{\Sigma} G \equiv 0$, which requires $\left|\nabla_{\Sigma} \rho\right| \equiv 1$ according to Lemma 1 . This can only happen when $\Sigma$ is totally geodesic.

We have tacitly assumed that $C \backslash\{p\}$ is immersed in $M$. Equation (3) may be proved in the general case either by analysis in singular coordinates or by approximation; we shall carry out an appropriate approximation argument at the end of the proof of the next proposition.

Proposition 2 (Gauss-Bonnet). Consider the geodesic cone $C=p \times \Gamma$ over an immersed $C^{2}$ curve $\Gamma$ in $\mathbf{S}_{+}^{n}, n \geq 2$.

(a) If $p \notin \Gamma$, then

$$
2 \pi \Theta_{C}(p)=\operatorname{Area}(C)-\int_{\Gamma} \vec{k} \cdot \nu_{C} d s .
$$

(b) If $p \in \Gamma$, then

$$
2 \pi \Theta_{C}(p)=\operatorname{Area}(C)-\int_{\Gamma} \vec{k} \cdot \nu_{C} d s-\pi .
$$

Proof. We first assume that $C \backslash\{p\}$ is immersed in $\mathbf{S}_{+}^{n}$.

Consider case (a), where $p \notin \Gamma$. By the Gauss-Bonnet formula on $C_{\varepsilon}$, for $\varepsilon$ less than the distance from $p$ to $\Gamma$,

$$
\int_{C_{\varepsilon}} K d A-\int_{\Gamma} \vec{k} \cdot \nu_{C} d s-\int_{C \cap \partial B_{\varepsilon}(p)} \vec{k} \cdot \nu_{C} d s=2 \pi \chi\left(C_{\varepsilon}\right) .
$$


where $\chi$ is the Euler number and $K$ is the intrinsic Gauss curvature of $C$. Since $C_{\varepsilon}$ is an immersed annulus, we have $\chi\left(C_{\varepsilon}\right)=0$. Now $C$ has principal curvature zero in the $\bar{\nabla} \rho$ direction, so the determinant of its second fundamental form vanishes, and by the Gauss equation, $K$ equals the sectional curvature $\bar{K}=1$ of the ambient $\mathbf{S}_{+}^{n}$.

Along $C \cap \partial B_{\varepsilon}(p), \nu_{C}=-\bar{\nabla} \rho$ and $\vec{k} \cdot \nu_{C} \equiv \cot \varepsilon$. Thus, we may compute

$$
\lim _{\varepsilon \rightarrow 0} \int_{C \cap \partial B_{\varepsilon}(p)} \vec{k} \cdot \nu_{C} d s=\lim _{\varepsilon \rightarrow 0}(\cot \varepsilon) L\left(C \cap \partial B_{\varepsilon}(p)\right)=2 \pi \Theta_{C}(p),
$$

so that formula (4) implies

$$
\operatorname{Area}(C)-\int_{\Gamma} \vec{k} \cdot \nu_{C} d s-2 \pi \Theta_{C}(p)=0
$$

which proves Proposition 2(a) when $C \backslash\{p\}$ is an immersion.

The proof of part (b) is analogous. However, when $p \in \Gamma$, for small $\varepsilon, C_{\varepsilon}$ is a topological disk, so that $\chi\left(C_{\varepsilon}\right)=1$. Also, the boundary of $C_{\varepsilon}$ consists of the arc $C \cap \partial B_{\varepsilon}(p)$ and the arc $\Gamma_{\varepsilon}:=\Gamma \backslash B_{\varepsilon}(p)$. For small $\varepsilon>0$, these arcs meet at two points forming exterior angles $\alpha(\varepsilon)$ and $\beta(\varepsilon)$. Equation (4) becomes

$$
\int_{C_{\varepsilon}} K d A-\int_{\Gamma_{\varepsilon}} \vec{k} \cdot \nu_{C} d s-\int_{C \cap \partial B_{\varepsilon}(p)} \vec{k} \cdot \nu_{C} d s+\alpha(\varepsilon)+\beta(\varepsilon)=2 \pi .
$$

Since $\Gamma$ is smooth, $\alpha(\varepsilon) \rightarrow \pi / 2$ and $\beta(\varepsilon) \rightarrow \pi / 2$ as $\varepsilon \rightarrow 0$, which yields

$$
\operatorname{Area}(C)-\int_{\Gamma} \vec{k} \cdot \nu_{C} d s-2 \pi \Theta_{C}(p)=\pi
$$

and Proposition 2(b) follows.

In general, the cone $C=p \circledast \Gamma$ need not be an immersion away from $p$. The problem arises exactly on the set $A \subset \Gamma$ where $\Gamma$ is tangent to the radial geodesic from $p$, that is, the unit tangent vector $\vec{T}$ coincides with $\pm \bar{\nabla} \rho$. Let us choose a $C^{1}$ mapping $\vec{T}_{\delta}$ from $\Gamma$ into the unit tangent bundle of $\mathbf{S}_{+}^{n} \backslash\{p\}$, which is $C^{1}$-close to $\vec{T}$ and transverse to the two sections $\pm \bar{\nabla} \rho$. The two sections $\pm \bar{\nabla} \rho$ define a codimension- $(n-1)$ submanifold of the total space of the unit tangent bundle. If $n \geq 3$, transversality means that $\vec{T}_{\delta}$ is disjoint from this submanifold. If $n=2$, we first embed $\mathbf{S}_{+}^{2}$ as a totally geodesic surface in $\mathbf{S}_{+}^{3}$, and then require transversality for $\vec{T}_{\delta}$. In order to ensure that $\vec{T}_{\delta}$ is the tangent vector to a closed curve $\Gamma_{\delta}$, we adjust $\vec{T}_{\delta}$ to satisfy the $n-1$ closure conditions, for small $\delta$. In the case $p \in \Gamma$, we may require $p \in \Gamma_{\delta}$. Then $p \times \Gamma_{\delta}$ satisfies formula (3), and formula (6) or (7), if $p \notin \Gamma$ or $p \in \Gamma$, respectively.

We claim that, since $\Gamma_{\delta} \rightarrow \Gamma$ in the $C^{2}$ norm, each term of equation (3), (6) or (7) is the limit, as $\delta \rightarrow 0$, of the corresponding quantity for $\Gamma_{\delta}$. To be precise, it should be observed that in general, the cone $C=p \circledast \Gamma$ is only $C^{1,1}$ up to the boundary $\Gamma$. Namely, the outward unit normal vector $\nu_{C}$ satisfies $\nu_{C} \cdot \bar{\nabla} \rho \geq 0$. For $q$ in the set $A \subset \Gamma, \nu_{C}(q)$ is nonunique; clearly, for $q_{\delta} \rightarrow$ $q, q_{\delta} \in \Gamma_{\delta}$, the normal vectors $\nu_{C_{\delta}}\left(q_{\delta}\right)$ need not converge. Nonetheless, the 
inward geodesic curvature $k=-\vec{k} \cdot \nu_{C}$ is well defined almost everywhere on $\Gamma$, since $\vec{k}=0$ almost everywhere on the problematic set $A$. Similarly, $\nu_{C} \cdot \bar{\nabla} \rho$ is well defined almost everywhere on $\Gamma$. Both $k$ and $\nu_{C} \cdot \bar{\nabla} \rho$ are pointwide limits almost everywhere of the corresponding quantities for $\Gamma_{\delta}$, which are uniformly bounded. The dominated convergence theorem now implies that formula (3), and either formula (6) or (7), hold for any $C^{2}$ curve $\Gamma \subset \mathbf{S}_{+}^{n}$.

Proof of Embedding Theorem 1. Let $\Sigma^{2}$ be a branched minimal surface in $\mathbf{S}_{+}^{n}$ whose boundary $\partial \Sigma=\Gamma$ is a $C^{2}$ Jordan curve satisfying the hypothesis (1):

$$
\mathcal{C}_{\text {tot }}(\Gamma):=\int_{\Gamma}|\vec{k}| d s \leq 4 \pi-\overline{\mathcal{A}}(\Gamma)
$$

Note that $\Sigma \subset \mathcal{H}_{\mathrm{cvx}}(\Gamma)$ by the maximum principle. To show that $\Sigma$ has no interior branch points and is embedded, it suffices to show that $\Theta_{\Sigma}(p)<2$ for all $p \in \Sigma(p \notin \Gamma)$.

Choose $p \in \Sigma$, and let $C=p \circledast \Gamma$ be the geodesic cone over $\Gamma$ with vertex $p$. If $\Sigma$ is totally geodesic, then it is the subset of a totally geodesic $\mathbf{S}_{+}^{2}$ bounded by the embedded curve $\Gamma \subset \mathbf{S}_{+}^{2}$, so $\Sigma$ is embedded. Otherwise, by Propositions 1 and 2(a), we have

$$
2 \pi \Theta_{\Sigma}(p)<2 \pi \Theta_{C}(p)=-\int_{\Gamma} \vec{k} \cdot \nu_{C} d s+\operatorname{Area}(C) .
$$

Since $p \in \mathcal{H}_{\mathrm{cvx}}(\Gamma)$, Area $(C)$ is less than or equal to the maximum cone area $\overline{\mathcal{A}}(\Gamma)$. But $-\vec{k} \cdot \nu_{C} \leq|\vec{k}|$, so hypothesis $(1)$ implies $\Theta_{\Sigma}(p)<2$, as required.

It remains to rule out boundary branch points (in the case $n=3$ of Theorem 2 , this would follow by well-known arguments from embeddedness in the interior, e.g. [GL].) If $p \in \Gamma$, then by Propositions 1 and $2(\mathbf{b})$, unless $\Sigma$ is totally geodesic, we have

$$
2 \pi \Theta_{\Sigma}(p)<2 \pi \Theta_{C}(p)=-\int_{\Gamma} \vec{k} \cdot \nu_{C} d s+\operatorname{Area}(C)-\pi .
$$

Using hypothesis (1) as before, we find that $\Theta_{\Sigma}(p)<3 / 2$. For a boundary branch point $p$ of order $k$, the density $\Theta_{\Sigma}(p) \geq(k+1) / 2$, and $k$ is even, by Lemma 2(a). This would imply that $\Theta_{\Sigma}(p) \geq 3 / 2$, which is impossible. We have shown that $\bar{\Sigma}$ is embedded.

\section{Embeddedness of Minimal Surfaces in Negatively Curved Spaces}

We now turn our attention to the case of nonpositive ambient sectional curvature. For a minimal surface in hyperbolic space, embeddedness may be proved in complete analogy to section 2 above, with $-\overline{\mathcal{A}}(\Gamma)$ replaced in hypothesis (1) by the infimum of areas of cones. However, unlike the case of $\mathbf{S}_{+}^{n}$, the nonpositively curved case can be significantly improved to permit variable sectional curvature, and the inequalities require only a nonpositive upper bound $-\kappa^{2}$ on ambient sectional curvature.

Thus, throughout this section we assume that $M$ is an $n$-dimensional complete, simply connected Riemannian manifold with sectional curvature bounded 
above by a nonpositive constant $-\kappa^{2}$. Let $\Gamma$ be a $C^{2}$ immersed curve in $M$. We define the (geodesic) cone $C=p \times \Gamma$ over $\Gamma$ with vertex $p$ as the union of the geodesic segments from $p$ to $q$, over all $q \in \Gamma$. Since the geodesic joining any two points of $M$ is unique and depends smoothly on its endpoints, $C \backslash\{p\}$ is the image of a $C^{2}$ mapping.

The main tool which will be added to the methods employed in Section 2 above is comparison with a metric $\widehat{g}$ of constant Gauss curvature $-\kappa^{2}$ on the geodesic cone $C$; see Definition 4 below. This metric was introduced by the first author in his study of isoperimetric inequalities on minimal surfaces ([C].)

Definition 3. Define the minimum cone area of $\Gamma$ as

$$
\mathcal{A}(\Gamma):=\inf _{p \in \mathcal{H}_{\mathrm{cvx}}(\Gamma)} \operatorname{Area}(p \circledast \Gamma) .
$$

Remark 1. A refinement of the methods of this paper would be to replace the convex hull of $\Gamma$ in Definitions 1 and 3 with the (usually) smaller meancurvature hull of $\Gamma$. This would allow Theorems 1 and 3 to be proved with slightly weaker hypotheses. The mean-curvature hull of a subset $S \subset M$ is defined as the intersection of the closures of $C^{2}$ open subsets of $M$ which contain $S$, have boundaries of nonnegative mean curvature (with respect to the inward unit normal), and which are members of a continuous exhaustion of $M$ by open subsets whose boundaries have nonnegative mean curvature. It follows that if $\Sigma$ is a branched minimal surface in $M$ with compact closure, then $\Sigma$ lies inside the mean-curvature hull of $\partial \Sigma$.

In this regard, it should be noted that Brickell and Hsiung actually proved the unknotting Theorem 4 for the special case when $M^{3}$ is the hyperbolic space of constant sectional curvature $-\kappa^{2}$, and the infimum of area is taken only over cones whose vertices lie on $\Gamma$ itself (see $[\mathrm{BH}]$.)

Theorem 3. Let $\Sigma^{2}$ be a branched minimal surface (of arbitrary topological type) in an $n$-dimensional complete, simply connected Riemannian manifold $M$ whose sectional curvature is bounded above by a nonpositive constant $-\kappa^{2}$. Write $\Gamma=\partial \Sigma$, which we assume to be a $C^{2}$ Jordan curve, i.e. a $C^{2}$ embedding of the circle $S^{1}$. If the total curvature of $\Gamma$ satisfies

$$
\mathcal{C}_{\text {tot }}(\Gamma):=\int_{\Gamma}|\vec{k}| d s \leq 4 \pi+\kappa^{2} \mathcal{A}(\Gamma)
$$

then $\bar{\Sigma}$ is an embedding.

We shall give the proof of Theorem 3 at the end of this section.

Theorem 3 implies a substantial extension of the Fáry-Milnor Theorem, which was proved for $\kappa=0$ in $[\mathrm{AB}]$ and $[\mathrm{S}]$. The proof of the following theorem is similar to the proof of Theorem 2 above.

Theorem 4. Let $\Gamma$ be a $C^{2}$ Jordan curve in a complete, simply connected Riemannian 3-manifold $M$ with sectional curvature $\leq-\kappa^{2}$. If the total curvature 
of $\Gamma$ satisfies

$$
\int_{\Gamma}|\vec{k}| d s \leq 4 \pi+\kappa^{2} \mathcal{A}(\Gamma)
$$

then $\Gamma$ is unknotted.

Example 2. This example shows that the hypothesis

$$
\mathcal{C}_{\text {tot }}(\Gamma) \leq 4 \pi+\kappa^{2} \mathcal{A}(\Gamma)
$$

of Theorems 3 and 4 is sharp.

Let $\Gamma_{0}$ be the double cover of the circle of radius $R$ in a totally geodesic $\mathbf{H}^{2} \subset \mathbf{H}^{3}$. Here $\mathbf{H}^{n}$ is the $n$-dimensional hyperbolic space of constant sectional curvature $-\kappa^{2}=-1$. In a similar fashion to Example 1, given any choice of positive integer $m$, the example is a one-parameter family of $(2,2 m+1)$-torus knots $\Gamma_{\eta}$ in $\mathbf{H}^{3}, \eta>0$, with $\Gamma_{\eta} \rightarrow \Gamma_{0}$ and with

$$
\mathcal{C}_{\text {tot }}\left(\Gamma_{\eta}\right)<4 \pi+\mathcal{A}\left(\Gamma_{\eta}\right)+\eta .
$$

In fact, $\Gamma_{0}$ has length $4 \pi \sinh R$, curvature $|\vec{k}| \equiv \operatorname{coth} R, \mathcal{C}_{\text {tot }}\left(\Gamma_{0}\right)=4 \pi \cosh R$, and $\mathcal{A}\left(\Gamma_{0}\right)=4 \pi(\cosh R-1)$.

We shall now present six results, in preparation for the proof of Theorem 3.

Write $G(r):=\log \tanh (\kappa r / 2)$ for the Green's function of the two-dimensional hyperbolic plane $\mathbf{H}^{2}\left(-\kappa^{2}\right)$ with Gauss curvature $\equiv-\kappa^{2}<0$, and $G(r):=\log r$ for $\mathbf{R}^{2}$, if $\kappa=0$. We compute $d G / d r=\kappa / \sinh \kappa r$ or $d G / d r=1 / r$, respectively. Choose a point $p \in M$, and define $\rho(x):=d(x, p)$, using the distance function $d(\cdot, \cdot)$ of $M$.

Lemma 3. Let $N^{2}$ be a two-dimensional manifold immersed in a complete, simply connected Riemannian manifold $M$ whose sectional curvature is bounded above by $-\kappa^{2}, \kappa \geq 0$. Then

(a) except at $p$,

$$
\triangle_{N} G(\rho) \geq 2 \kappa^{2} \frac{\cosh \kappa \rho}{\sinh ^{2} \kappa \rho}\left(1-\left|\nabla_{N} \rho\right|^{2}\right)+\kappa \frac{d \rho(\vec{H})}{\sinh \kappa \rho} \text { in case } \kappa>0,
$$

and

$$
\triangle_{N} G(\rho) \geq \frac{2}{\rho^{2}}\left(1-\left|\nabla_{N} \rho\right|^{2}\right)+\frac{d \rho(\vec{H})}{\rho} \text { in case } \kappa=0,
$$

where $\vec{H}$ is the mean curvature vector of $N$.

(b)

$$
\triangle_{N} \log (1+\cosh \kappa \rho) \geq \kappa^{2}+\kappa \tanh (\kappa \rho / 2) d \rho(\vec{H}) \text { in case } \kappa>0,
$$

and

$$
\triangle_{N} \rho^{2} \geq 4+2 \rho d \rho(\vec{H}) \text { in case } \kappa=0
$$


Proof. By the Hessian comparison theorem, the Hessian of the distance function $\rho$ of $M$ satisfies

$$
\bar{\nabla}^{2} \rho \geq \kappa \operatorname{coth} \kappa \rho(g-\bar{\nabla} \rho \otimes \bar{\nabla} \rho) \text { for } \kappa>0, \text { and } \bar{\nabla}^{2} \rho^{2} \geq 2 g \text { for } \kappa=0,
$$

where $g$ is the metric tensor of $M$ (see [SY], p.4).

As in the proof of Lemma 1, after applying the trace formula, this inequality leads us to the conclusion of part (a).

For the proof of part (b), we again use the trace formula and note that

$$
\bar{\nabla}^{2} \log (1+\cosh \kappa \rho) \geq \frac{\kappa^{2}}{1+\cosh \kappa \rho}[\cosh \kappa \rho \cdot g+(1-\cosh \kappa \rho) \bar{\nabla} \rho \otimes \bar{\nabla} \rho] \text { for } \kappa>0 .
$$

For a 2-dimensional immersed Lipschitz submanifold, or a branched surface, $N \subset M$ and a point $q \in M$, we define the density of $N$ at $q$ to be the limit

$$
\Theta_{N}(q):=\lim _{\varepsilon \rightarrow 0} \frac{\operatorname{Area}\left(N \cap B_{\varepsilon}(q)\right)}{\pi \varepsilon^{2}}
$$

as in definition (2) above. As observed in section 2 above, if $N$ is a smoothly immersed submanifold of $M$ and has a self-intersection at $p \in M$, then $\Theta_{N}(p) \geq$ 2. Further, if $p$ is an interior branch point of $N$ of order $k$, then $\Theta_{N}(p) \geq k+1$; at a boundary branch point, $\Theta_{N}(p) \geq(k+1) / 2$.

Let $\Gamma$ be a $C^{2}$ immersed closed curve in $M$. Choose $p \in M$. If $\Sigma^{2}$ is a branched minimal surface in $M$ with boundary $\partial \Sigma=\Gamma$, and $C$ is the cone $p * \Gamma$ over $p$, then the key ingredient in the proof of Theorem 3 is to give an upper bound of $\Theta_{\Sigma}(p)$ by $\Theta_{C}(p)$. Unfortunately this is impossible unless $M$ is rotationally symmetric about $p$. To get around this difficulty we need to define a constant-curvature metric $\widehat{g}$ on $C$ as follows.

Definition 4. Let $\widehat{g}$ be a new metric on $C$ with constant Gauss curvature $-\kappa^{2}$ such that the distance from $p$ remains the same as in the original metric $g$, and so does the arclength element of $\Gamma$. More precisely, every geodesic from $p$ under $g$ remains a geodesic of equal length under $\widehat{g}$, the length of any arc of $\Gamma$ remains the same, and the angles between the tangent vector to $\Gamma$ and the geodesic from $p$ remain unchanged.

We shall write $\widehat{C}$ for the two-dimensional Riemannian manifold $(C, \widehat{g})$, which is singular at $p$. In order to construct $\widehat{C}$, we may start with an arc-length parameter $s$ along $\Gamma$. Let $r(s)$ be the distance in $C$ from the corresponding point of $\Gamma$ to $p$. Then choose a point $\widehat{p} \in \mathbf{H}^{2}\left(-\kappa^{2}\right)$, and let a curve $\widehat{\Gamma}$ locally isometric to $\Gamma$ be traced out in $\mathbf{H}^{2}\left(-\kappa^{2}\right)$ so that the distance from $\widehat{p}$ equals $r(s)$. Let $\widehat{C}=\widehat{p} * \widehat{\Gamma}$, which may be in a covering of $\mathbf{H}^{2}\left(-\kappa^{2}\right)$ branched over $\widehat{p}$, and finally glue $\widehat{C}$ along the geodesic segments from $\widehat{p}$ to the initial and final points (cf. [C], p. 211.) Note that the angle between two geodesics at $p$ becomes larger under $\widehat{g}$, as we shall see in Proposition 5 below. 


\section{Corollary 2.}

(a) If $\Sigma^{2}$ is a branched minimal surface in $M$, then $G(\rho)$ is subharmonic on $\Sigma$.

(b) If $\widehat{C}$ is the cone $p * \partial \Sigma$ over the pole $p$ of the distance function $\rho$ in $M$ with the metric $\widehat{g}$ of Gauss curvature $\equiv-\kappa^{2}$, then $G(\rho)$ is harmonic on $\widehat{C}$, except at $p$.

(c) Further, on $\widehat{C}$

$$
\begin{gathered}
\triangle_{\hat{C}} \log (1+\cosh \kappa \rho)=\kappa^{2} \text { for } \kappa>0, \text { and } \\
\triangle_{\hat{C}} \rho^{2}=4 \text { for } \kappa=0 .
\end{gathered}
$$

Proof.

(a) On $\Sigma$, the mean curvature vector of $\Sigma$ vanishes and $\left|\nabla_{\Sigma} \rho\right| \leq 1$, hence $\triangle_{\Sigma} G(\rho) \geq 0$, except at $p$, according to Lemma 3(a). Near $p$, we argue as in the proof of Corollary 1.

(b) On the cone $\widehat{C}$, however, we apply Lemma 3(a) with $M=N=\widehat{C}$, so that $\vec{H} \equiv 0$ and $\left|\nabla_{\hat{C}} \rho\right| \equiv 1$. Moreover constancy of the Gauss curvature on $\widehat{C}$ forces all the inequalities in the proof of Lemma 3(a) to become equality and consequently $\triangle_{\hat{C}} G(\rho) \equiv 0$.

(c) Similarly for part (c).

Remark 2. The following four propositions treat the cone $C=p \times \Gamma$. In the proof of each, it is convenient to assume that the cone is immersed except at $p$. This implies that $\widehat{C} \backslash\{p\}$ is a smooth two-dimensional manifold with Gauss curvature $\widehat{K} \equiv-\kappa^{2}$. This assumption entails no loss of generality, since, as a curve in $M, \Gamma$ is the $C^{2}$ limit of closed curves $\Gamma_{\delta}$ with the property that $p * \Gamma_{\delta}$ is immersed except at $p$. Specifically, the geodesic curvatures $k$ and $\widehat{k}$ considered below, and the normal derivative $\nu_{C} \cdot \bar{\nabla} \rho$ of $\rho$, are the pointwise limits almost everywhere of the corresponding quantities for $\Gamma_{\delta}$. This may be proven as at the end of the proof of Proposition 2 above.

Proposition 3 (Density Comparison). Let $\Sigma^{2}$ be a branched minimal surface in an $n$-dimensional simply connected Riemannian manifold $M$ with sectional curvature $\leq-\kappa^{2}$. If $\widehat{C}$ is as in Definition 4 above, then $\Theta_{\Sigma}(p)<\Theta_{\hat{C}}(p)$ unless $\Sigma$ is totally geodesic with constant Gauss curvature $-\kappa^{2}$.

Proof. By Corollary 2, we have $\triangle_{\Sigma} G(\rho) \geq 0$ and $\triangle_{\hat{C}} G(\rho) \equiv 0$, where, as above, $G(\rho(x)):=\log \tanh (\kappa \rho(x) / 2)$ and $\rho(x):=d_{M}(x, p)$ or $d_{\hat{C}}(x, p)$ respectively. For small $\varepsilon>0$, write $\widehat{C}_{\varepsilon}:=\widehat{C} \backslash B_{\varepsilon}(p)$ and $\Sigma_{\varepsilon}:=\Sigma \backslash B_{\varepsilon}(p)$, where $B_{\varepsilon}(p)$ denotes the geodesic ball in $M$ of radius $\varepsilon$ and center $p$. Then the boundary of $\Sigma_{\varepsilon}$ is $\Gamma \cup\left(\Sigma \cap \partial B_{\varepsilon}(p)\right)$. (The component $\Sigma \cap \partial B_{\varepsilon}(p)$ may be empty.) Let $\nu_{\Sigma}$ be the outward unit normal vector tangent to $\Sigma_{\varepsilon}$ at $\partial \Sigma_{\varepsilon}$. Then

$0 \leq \int_{\Sigma_{\varepsilon}} \triangle_{\Sigma} G(\rho) d A=\int_{\partial \Sigma_{\varepsilon}} \nu_{\Sigma} \cdot \bar{\nabla} G d s=\int_{\Sigma \cap \partial B_{\varepsilon}(p)} \kappa \frac{\nu_{\Sigma} \cdot \bar{\nabla} \rho}{\sinh \kappa \varepsilon} d s+\int_{\Gamma} \kappa \frac{\nu_{\Sigma} \cdot \bar{\nabla} \rho}{\sinh \kappa \rho} d s$. 
Along the small boundary component $\Sigma \cap \partial B_{\varepsilon}(p)$, as $\varepsilon \rightarrow 0, \nu_{\Sigma} \cdot \bar{\nabla} \rho \rightarrow-1$ uniformly, and

$$
\kappa \frac{L\left(\Sigma \cap \partial B_{\varepsilon}(p)\right)}{2 \pi \sinh \kappa \varepsilon} \rightarrow \Theta_{\Sigma}(p)
$$

Let $\nu_{C}$ be the outward unit normal vector tangent to $C$ along its boundary. Then it should be noted that

$$
\nu_{\Sigma} \cdot \bar{\nabla} \rho \leq \nu_{C} \cdot \bar{\nabla} \rho \text { along } \Gamma .
$$

Thus, we find that the inequality above implies

$$
2 \pi \Theta_{\Sigma}(p) \leq \int_{\Gamma} \kappa \frac{\nu_{C} \cdot \bar{\nabla} \rho}{\sinh \kappa \rho} d s
$$

Note here that $\nu_{C}$, considered as a tangent vector to $C$, is also the outward unit normal vector in the metric $\widehat{g}$. Along the intrinsic distance sphere $\partial \widehat{B}_{\varepsilon}(p) \subset$ $\widehat{C},-\nabla \rho$ is the outward unit normal vector tangent to $\widehat{C}_{\varepsilon}$. Hence by Corollary 2(b), assuming $C \backslash\{p\}$ is immersed, as $\varepsilon \rightarrow 0$,

$$
0=\int_{\hat{C}_{\varepsilon}} \triangle_{\hat{C}} G(\rho) d A \rightarrow-2 \pi \Theta_{\hat{C}}(p)+\int_{\Gamma} \kappa \frac{\nu_{C} \cdot \nabla \rho}{\sinh \kappa \rho} d s .
$$

See Remark 2 for the non-immersed case. Therefore, by inequality (10),

$$
2 \pi \Theta_{\hat{C}}(p)=\int_{\Gamma} \kappa \frac{\nu_{C} \cdot \bar{\nabla} \rho}{\sinh \kappa \rho} d s \geq 2 \pi \Theta_{\Sigma}(p),
$$

which is the desired estimate.

If equality holds, then $\triangle_{\Sigma} G \equiv 0$, which requires $\left|\nabla_{\Sigma} \rho\right| \equiv 1$ according to Lemma 3. But this means that $\Sigma$ is a cone over $p$, as well as being minimal, which can only occur when $\Sigma$ is totally geodesic. Moreover, $\triangle_{\Sigma} G \equiv 0$ now implies that $\triangle_{\Sigma} \rho \equiv \kappa \operatorname{coth} \kappa \rho$, which, along with $K_{\Sigma} \leq-\kappa^{2}$, implies that $\Sigma$ has constant Gauss curvature $K_{\Sigma} \equiv-\kappa^{2}$.

Proposition 4 (Geodesic Curvature Comparison). Let $\Gamma$ be a $C^{2}$ curve in $M^{n}$, a manifold with sectional curvatures $\leq-\kappa^{2}$, and let $C$ be the cone $p \times \Gamma$. If $\widehat{C}$ is the cone $C$ with the constant curvature metric $\widehat{g}$, as in Definition 4 above, then $k(q) \geq \widehat{k}(q)$ for almost all $q \in \Gamma$, where $k$ and $\widehat{k}$ denote the inward geodesic curvatures of $\Gamma$ in $C$ and $\widehat{C}$, respectively.

Proof. We first assume that $C \backslash\{p\}$ is immersed. For $\rho_{0}>0$, let $\Gamma_{0}=C \cap$ $\partial B_{\rho_{0}}(p)$, and let $k_{0}$ be the geodesic curvature of $\Gamma_{0}$ in $C$. Also, let $\widehat{k}_{0}$ be the geodesic curvature of $\Gamma_{0}$ in $\widehat{C}$. To estimate $k_{0}$ and $\widehat{k}_{0}$ let us define $V(\widehat{V}$, respectively) to be a Jacobi field in $C$ ( $\widehat{C}$, respectively) along the unit-speed geodesic $\gamma$ from $p$ to $q \in \Gamma$, satisfying

$$
V(p)=\widehat{V}(p)=0 \text { and } V \perp \dot{\gamma}, \widehat{V} \perp \dot{\gamma}
$$

For each $q \in \Gamma$, since $g=\widehat{g}$ along $\Gamma$, we may also impose the boundary conditions

$$
V(q)=\widehat{V}(q),|V(q)|=|\widehat{V}(q)|=1,
$$


thereby determining $V$ and $\widehat{V}$ uniquely, since $K$ and $\widehat{K}$, the Gauss curvatures of $C$ and $\widehat{C}$ respectively, are nonpositive. In fact, $V=\widehat{V}$ as vector fields on $C \backslash\{p\}$. $V$ and $\widehat{V}$ satisfy the Jacobi equations

$$
\nabla_{\dot{\gamma}} \nabla_{\dot{\gamma}} V=R(\dot{\gamma}, V) \dot{\gamma} \text { and } \widehat{\nabla}_{\dot{\gamma}} \widehat{\nabla}_{\dot{\gamma}} \widehat{V}=\widehat{R}(\dot{\gamma}, \widehat{V}) \dot{\gamma}
$$

where $\nabla, \widehat{\nabla}$ denote the connections for the metrics $g, \widehat{g}$ respectively, while $R, \widehat{R}$ denote the Riemann curvature tensors of $g$ and $\widehat{g}$, respectively. Write $f(t)=$ $\|V(\gamma(t))\|$, and similarly $\widehat{f}(t)=\|\widehat{V}(\gamma(t))\|$, where the norms are measured using $g$ and $\widehat{g}$, respectively. Since $C$ and $\widehat{C}$ have dimension 2, equations (13) are equivalent to the scalar Jacobi equations

$$
f^{\prime \prime}(t)+K(\gamma(t)) f(t)=0, \quad \widehat{f}^{\prime \prime}(t)+\widehat{K}(\gamma(t)) \widehat{f}(t)=0 .
$$

By the Gauss equation we have

$$
K=R_{M}(\dot{\gamma}, V, V, \dot{\gamma}) /\|V\|^{2}+\operatorname{det}(B),
$$

where $R_{M}$ is the Riemann curvature tensor of $M$ and $B$ is the second fundamental form of $C$ in $M$. Since $C$ is a cone, we have $\operatorname{det}(B)=0$, and it follows that $C$ has Gauss curvature

$$
K \leq-\kappa^{2} .
$$

We next compute $k_{0}$ and $\widehat{k}_{0}$. Extend $V$ and $\widehat{V}$ as normal Jacobi fields along all radial geodesics from $p$. Also, let $W$ be the unit vector field which is tangent to the radial geodesics. Then $[V, W] \equiv 0$ and $\langle V, W\rangle \equiv 0$. Similarly, $[\widehat{V}, W] \equiv 0$ and $\langle\widehat{V}, W\rangle \equiv 0$. Then

$$
\|V\|^{2} k_{0}=-\left\langle\bar{\nabla}_{V} V, W\right\rangle=\left\langle V, \bar{\nabla}_{V} W\right\rangle=\left\langle V, \bar{\nabla}_{\dot{\gamma}} V\right\rangle=\dot{\gamma}\left(\|V\|^{2}\right) / 2=f^{\prime}(t) f(t) .
$$

Thus $k_{0}(\gamma(t))=f^{\prime}(t) / f(t)$. Similarly, we compute $\widehat{k}_{0}(\gamma(t))=\widehat{f}^{\prime}(t) / \widehat{f}(t)$. As is well known, the scalar Jacobi equations (14) are equivalent to the Riccati equations

$$
k_{0}^{\prime}(\gamma(t))+k_{0}(\gamma(t))^{2}=-K(\gamma(t)) \geq \kappa^{2}
$$

and

$$
\widehat{k}_{0}^{\prime}(\gamma(t))+\widehat{k}_{0}(\gamma(t))^{2}=-\widehat{K}(\gamma(t))=\kappa^{2} .
$$

It follows that the difference satisfies a homogeneous linear differential inequality

$$
\left(k_{0}-\widehat{k}_{0}\right)^{\prime}+\left(k_{0}+\widehat{k}_{0}\right)\left(k_{0}-\widehat{k}_{0}\right)=-K+\widehat{K} \geq 0 .
$$

Meanwhile, $k_{0}-\widehat{k}_{0}=\left(f^{\prime} \widehat{f}-\widehat{f}^{\prime} f\right) /(\widehat{f} f) \rightarrow 0$ as $t \rightarrow 0$, as follows from L'Hospital's rule using the equations (14). Therefore

$$
f^{\prime} / f-\widehat{f}^{\prime} / \widehat{f}=k_{0}-\widehat{k}_{0} \geq 0 .
$$

We are now in a position to compare the respective inward geodesic curvatures $k$ and $\widehat{k}$ of $\Gamma$. Write $T=(V / f) \cos \varphi-W \sin \varphi$ for the unit tangent vector to $\Gamma: T$ has unit length with respect to either metric $g$ or $\widehat{g}$. Then $\nabla_{T} T=-k \nu_{C}$ and $\widehat{\nabla}_{T} T=-\widehat{k} \nu_{C}$, where $\nu_{C}=(V / f) \sin \varphi+W \cos \varphi$ is the outward unit normal vector to $\Gamma$, with respect to either metric, and $\cos \varphi \geq 0$. We compute $\nabla_{W} W=\nabla_{W}(V / f)=0, \nabla_{V / f}(V / f)=-k_{0} W$ and $\nabla_{V} W=k_{0} V$. It follows in 
a straightforward fashion that $-k \nu_{C}=\nabla_{T} T=-k_{0} \nu_{C} \cos \varphi-\nu_{C} T(\varphi)$. Thus $k=k_{0} \cos \varphi+T(\varphi)$, and similarly $\widehat{k}=\widehat{k}_{0} \cos \varphi+T(\varphi)$. Hence

$$
k-\widehat{k}=\left(k_{0}-\widehat{k}_{0}\right) \cos \varphi \geq 0 .
$$

Remark 2 now implies that $k \geq \widehat{k}$ almost everywhere in the general case where $C \backslash\{p\}$ need not be immersed.

Remark 3. The proof of Proposition 4 holds more generally, for any two metrics $g, \widehat{g}$ on a cone which have the same unit-speed geodesics from the vertex, agree at the boundary, and whose Gaussian curvatures satisfy $K \leq \widehat{K}$.

Proposition 5 (Density and Area Comparison). Let $\Gamma$ be a $C^{2}$ curve in $M^{n}$, and let $C=p \times \Gamma$, as in Proposition 4. If $\widehat{C}$ is the cone $C$ with the constant curvature metric $\widehat{g}$, as in Definition 4 above, then the densities $\Theta_{C}(p) \leq \Theta_{\hat{C}}(p)$ and the areas $\operatorname{Area}(C) \leq \operatorname{Area}(\widehat{C})$.

Proof. The inequality (15) above implies that $f(t) / \widehat{f}(t)$ is increasing. Recalling the normalization $f=\widehat{f}$ at each $q \in \Gamma$ and $f=\widehat{f}=0$ at $p$, we see that $f(t) \leq \widehat{f}(t)$ along $\gamma, f^{\prime} \geq \widehat{f}^{\prime}$ at $q$, and $f^{\prime} \leq \widehat{f}^{\prime}$ at $p$. Note that Area $(C)$ and Area $(\widehat{C})$ may be written as the same double integral with respective integrands $f$ and $\widehat{f}$.

Remark 4. We note here an interesting inequality, related to Proposition 5 above, although we will not need it in this paper:

$$
\operatorname{Area}(\Sigma) \leq \operatorname{Area}(\widehat{C}) \text {. }
$$

The proof follows analogously to Proposition 3, using Lemma 3(b) and Corollary 2.

Proposition 6 (Gauss-Bonnet).

(a) For any geodesic cone $\widehat{C}=p \times \Gamma, p \notin \Gamma$, with constant curvature $-\kappa^{2}$ over an immersed $C^{2}$ curve $\Gamma$ in $M^{n}, n \geq 2$,

$$
2 \pi \Theta_{\widehat{C}}(p)+\kappa^{2} \operatorname{Area}(\widehat{C})=\int_{\Gamma} \widehat{k} d s
$$

where $\widehat{k}$ is the geodesic curvature of $\Gamma$ in $\widehat{C}$.

(b) If $p \in \Gamma$, then

$$
2 \pi \Theta_{\hat{C}}(p)+\kappa^{2} \operatorname{Area}(\widehat{C})=\int_{\Gamma} \widehat{k} d s-\pi
$$

Proof. (a) Consider $p \notin \Gamma$. By the Gauss-Bonnet formula on $\widehat{C}_{\varepsilon}:=\widehat{C} \backslash B_{\varepsilon}(p)$,

$$
\int_{\hat{C}_{\varepsilon}} \widehat{K} d A+\int_{\Gamma} \widehat{k} d s+\int_{\hat{C} \cap \partial B_{\varepsilon}(p)} \widehat{k} d s=2 \pi \chi\left(\widehat{C}_{\varepsilon}\right)=0
$$

where $\widehat{K} \equiv-\kappa^{2}$ is the intrinsic Gauss curvature of $\widehat{C}_{\varepsilon}$. Since $\widehat{C}_{\varepsilon}$ is an immersed annulus, the Euler number $\chi\left(\widehat{C}_{\varepsilon}\right)=0$. 
The geodesic curvature of $\widehat{C} \cap \partial B_{\varepsilon}(p)$ is the negative of the curvature of $\partial B_{\varepsilon}(p)$ as a curve in $\mathbf{H}^{2}\left(-\kappa^{2}\right)$, namely, $-\kappa \operatorname{coth} \kappa \varepsilon$. Thus,

$$
\begin{aligned}
\lim _{\varepsilon \rightarrow 0} \int_{\hat{C} \cap \partial B_{\varepsilon}(p)} \widehat{k} d s & =-\lim _{\varepsilon \rightarrow 0}(\kappa \operatorname{coth} \kappa \varepsilon) L\left(\widehat{C} \cap \partial B_{\varepsilon}(p)\right) \\
& =-\lim _{\varepsilon \rightarrow 0}(\cosh \kappa \varepsilon) 2 \pi \Theta_{\hat{C}}(p)=-2 \pi \Theta_{\hat{C}}(p) .
\end{aligned}
$$

Since $\operatorname{Area}\left(\widehat{C}_{\varepsilon}\right) \rightarrow \operatorname{Area}(\widehat{C})$, the Gauss-Bonnet formula (16) now implies

$$
-\kappa^{2} \operatorname{Area}(\widehat{C})+\int_{\Gamma} \widehat{k} d s-2 \pi \Theta_{\hat{C}}(p)=0,
$$

which proves Proposition 6(a) when $C \backslash\{p\}$ is an immersion. The general case follows from Remark 2. The proof of (b) is analogous to (a) and Proposition $2(\mathrm{~b})$.

Proof of Embedding Theorem 3. Let $\Sigma^{2}$ be a branched minimal surface in $M$ whose boundary $\partial \Sigma=\Gamma$ is a $C^{2}$ Jordan curve satisfying the hypothesis (8):

$$
\mathcal{C}_{\text {tot }}(\Gamma):=\int_{\Gamma}|\vec{k}| d s \leq 4 \pi+\kappa^{2} \mathcal{A}(\Gamma)
$$

where $-\kappa^{2}$ is an upper bound on sectional curvatures of the ambient manifold $M$. We need to show that $\bar{\Sigma}$ has no branch points and is embedded. Thus, it will suffice to show that $\Theta_{\Sigma}(p)<2$ at all $p \in M \backslash \Gamma$ and that $\Theta_{\Sigma}(p)<3 / 2$ at $p \in \Gamma$.

Consider any $p \in \Sigma \backslash \Gamma$, and let $C=p \circledast \Gamma$ be the geodesic cone over $\Gamma$ with vertex $p$. If $\Sigma$ is totally geodesic, then $\Sigma$ is embedded, since there are no compact totally geodesic surfaces and no geodesic loops in $M$. Otherwise, by Proposition 3 and Proposition 6(a), we have

$$
2 \pi \Theta_{\Sigma}(p)<2 \pi \Theta_{\hat{C}}(p)=\int_{\Gamma} \widehat{k} d s-\kappa^{2} \operatorname{Area}(\widehat{C}) .
$$

Recall that $\Sigma \subset \mathcal{H}_{\mathrm{cvx}}(\Gamma)$. Hence Proposition 5 implies that Area $(\widehat{C})$ is at least equal to the minimum cone area $\mathcal{A}(\Gamma)$, and since $\widehat{k} \leq k \leq|\vec{k}|$ almost everywhere along $\Gamma$ by Proposition 4, we find

$$
2 \pi \Theta_{\Sigma}(p)<\mathcal{C}_{\text {tot }}(\Gamma)-\kappa^{2} \mathcal{A}(\Gamma) .
$$

Therefore, hypothesis (8) implies $\Theta_{\Sigma}(p)<2$. If $p \in \Gamma$, apply Proposition 6(b) to show $\Theta_{\Sigma}(p)<3 / 2$. Then, as in the proof of Theorem 1, the embedded character of $\bar{\Sigma}$ follows.

\section{Acknowledgement}

The second author would like to thank Brian White for his pellucid private introduction to $[\mathrm{EWW}]$. 


\section{References}

[AB] S. Alexander, R. L. Bishop, The Fary-Milnor theorem in Hadamard manifolds, Proc. Amer. Math. Soc. 126 (1998), 3427-3436.

[AS] F. J. Almgren Jr., L. Simon, Existence of embedded solutions of Plateau's problem, Ann. Scuola Norm. Sup. Pisa Cl. Sci. (4) 6 (1979), 447-495.

[A] H.W. Alt, Verzweigungspunkte von H-Flächen. I, Math. Z. 127 (1972), 333-362; II, Math. Ann. 201 (1973), 33-56.

[BH] F. Brickell, C. C. Hsiung, The total absolute curvature of closed curves in Riemannian manifolds, J. Differential Geom. 9 (1974), 177-193.

[C] J. Choe, The isoperimetric inequality for minimal surfaces in a Riemannian manifold, J. Reine Angew. Math. 506 (1999), 205-214.

[CG1] J. Choe, R. Gulliver, The sharp isoperimetric inequality for minimal surfaces with radially connected boundary in hyperbolic space, Invent. Math. 109 (1992), 495-503.

[CG2] - Isoperimetric inequalities on minimal submanifolds of space forms, Manuscripta Math. 77 (1992), 169-189.

[D] J. Douglas, Solution of the problem of Plateau, Trans. Amer. Math. Soc. 33 (1931), 263-321.

[EWW] T. Ekholm, B. White, D. Wienholtz, Embeddedness of minimal surfaces with total

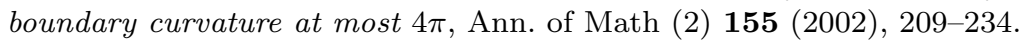

[F] M. I. Fáry, Sur la courbure totale d'une courbe gauche faisant un noeud, Bull. Soc. Math. France 77 (1949), 128-138.

[Fed] H. Federer, Geometric measure theory, Die Grundlehren der mathematischen Wissenschaften, Band 153 Springer-Verlag, New York, 1969.

[G] R. Gulliver, Regularity of minimizing surfaces of prescribed mean curvature, Ann. of Math. (2) 97 (1973), 275-305.

[GL] R. Gulliver, F. D. Lesley, On boundary branch points of minimizing surfaces, Arch. Rational Mech. Anal. 52 (1973), 20-25.

[GS] R. Gulliver, J. Spruck, On embedded minimal surfaces, Annals of Math. 103 (1976), $331-347$.

[HS] R. Hardt, L. Simon, Boundary regularity and embedded solutions for the oriented Plateau problem, Ann. of Math. (2) 110 (1979), 439-486.

$[\mathrm{HH}] \quad$ E. Heinz, S. Hildebrandt, Some remarks on minimal surfaces in Riemannian manifolds, Comm. Pure Appl. Math. 23 (1970), 371-377.

[MY] W. H. Meeks III, S.-T. Yau, The classical Plateau problem and the topology of threedimensional manifolds. The embedding of the solution given by Douglas-Morrey and an analytic proof of Dehn's lemma, Topology 21 (1982), 409-442.

[M] J. Milnor, On the total curvature of knots, Ann. of Math. (2) 52 (1950), 248-257.

[Mo] C.B. Morrey, Jr., The problem of Plateau on a Riemannian manifold, Annals of Math. 49 (1948), 807-851.

[N1] J.C.C. Nitsche, The boundary behavior of minimal surfaces. Kellogg's theorem and branch points on the boundary, Invent. Math. 8 (1969), 313-333.

[N2] _ A new uniqueness theorem for minimal surfaces, Arch. Rational Mech. Anal. 52 (1973), 319-329.

[N3] Lectures on minimal surfaces, Vol. 1. Cambridge University Press, Cambridge, 1989.

[R1] T. Radó, The problem of least area and the problem of Plateau, Math. Z. 32 (1930), 763-796.

[R2] - On the problem of Plateau. Subharmonic functions, Reprint. Springer-Verlag, New York-Heidelberg, 1971.

[SY] R. Schoen, S.-T. Yau, Lectures on differential geometry, Conference Proceedings and Lecture Notes in Geometry and Topology, I. International Press, Cambridge, MA, 1994. 
[S] C. Schmitz, The theorem of Fáry and Milnor for Hadamard manifolds, Geom. Dedicata 71 (1998), 83-90.

[TT] F. Tomi, A. J. Tromba, Extreme curves bound embedded minimal surfaces of the type of the disc, Math. Z. 158 (1978), 137-145.

Department of Mathematics, Seoul National University, Seoul, 151-742, Korea.

E-mail address: choe@math.snu.ac.kr

School of Mathematics, 127 Vincent Hall, University of Minnesota, Minneapolis, MN 55414, U.S.A.

E-mail address: gulliver@math.umn.edu 